\title{
Flying to Mars
}

\author{
By SONG Jianlan (Staff Reporter)
}

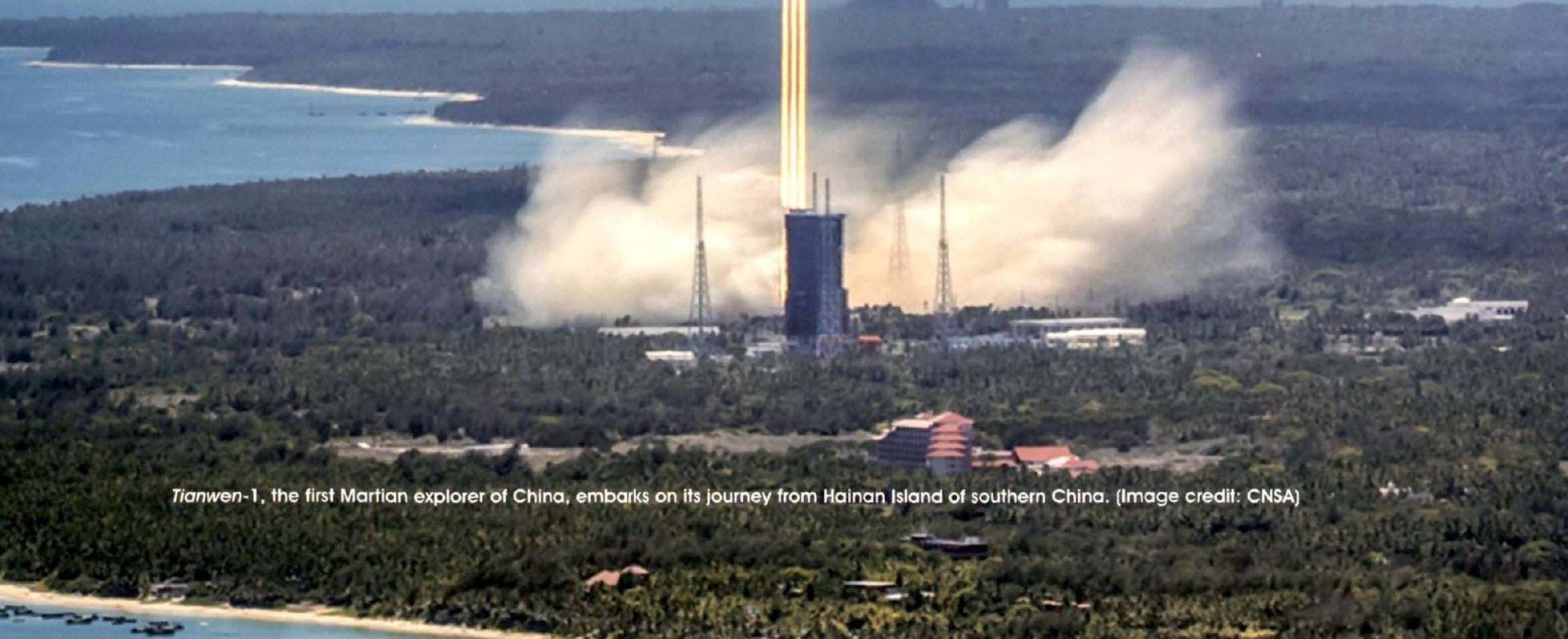


A 12:+1 (GMT+8) July 2:3. Tianwen-1. the first Mars explorer of China. embarked on its flight fom the Wenchang Satellite Launching Center in China's southern province of Hainan - with the help of Long March-5 Yt, a carrier rocket in the new high-thrust family developed by Chinese scientists and engineers. After flying for 2.156 seconds. the probe successfully separated from the rocket and entered the preset Earth-Mars transfer orbit. signaling the initial success of the launching.

After a long flight of about seven months, it is expected to reach Mars around February 2021, and start scientific observations from April 2021 .

While flying to the red planet, the probe has to beat the harsh conditions in outer space and meanwhile keep working. Thanks to advanced multi-object interferometry techniques. the payload can meet the tough requirements to transmit massive data over the long distance back to the Earth. and accurately measure and predict the orbit for the explorer with very little time delay.

Equipped with $1: 3$ devices and two data processors. Tianuen-1 consists of an orbiter and a Mars rover. Upon its arrival to Mars, the orbiter will circle Mars for global, extensive survey, and later send the rover to softly land on the Martian surface and conduct in-situ investigations of high accuracy and resolution at some locations of scientific interest. When the rover working on the Martian surface, the orbiter will stay in-orbit for relay communication and coordination.
Taken from the title of a poem by QU Yuan. an ancient Chinese poet living over two thousand vears ago, the name "Tianuen" means "questioning Heaven" and expresses Chinese scientists" aspirations to pursue planetary science. Mars represents a major focus of China s deep space exploration. which has gone beyond the Earth-Moon system with the start of the Mars exploration. Announced in 2016 as part of the country's layout for deep space exploration in the future decades, this grand program extends into 20.50 and consists of three stages, respectively focused on orbiting. landing and roving. involving sample return and a prospect research station on Mars. The data and materials collected will help answer questions like the origin and evolution of our solar system. the existence of extraterrestrial life, and whether or not human habitation is possible on this planet.

Specifically. the mission Tiamuen-1 will map the morphology and geological structure of Mars. investigate the characteristics of its surface soil and the distribution of water/ice. Also, the mission will analyze the composition of its surface materials, measure its ionosphere, its climate and enviromment from the surface. and understand its physical fields. including electromagnetic and gravitational fields, and its internal structure.

For China s Mars Exploration Program, the Chinese Academy of Sciences (CAS) proposed and verified the scientific objectives and layout for its payload allocation.

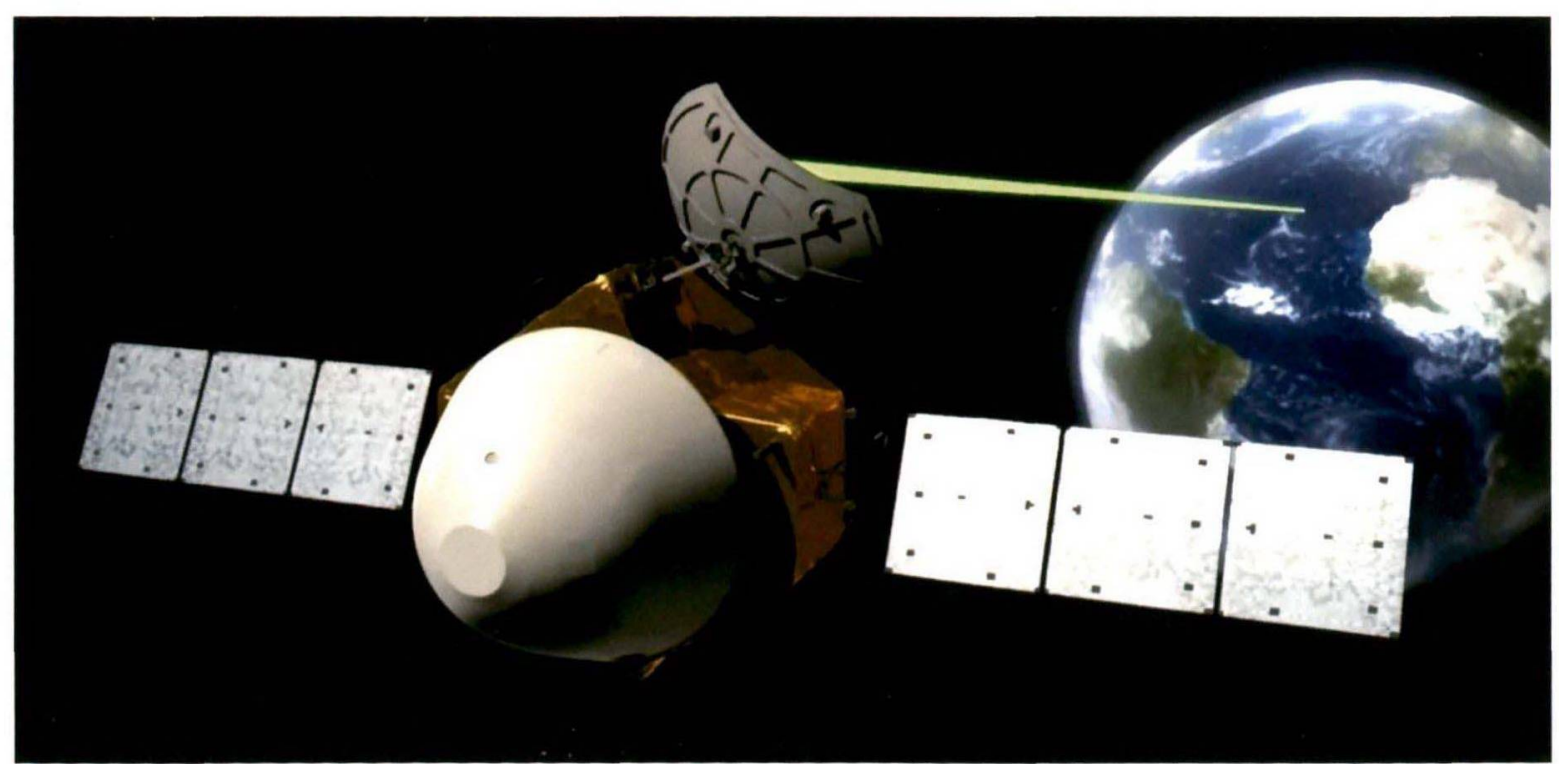

Illustrated on the front of the probe is the lander-rover complex, and in the rear the orbiter. (Image credit: CNSA) 


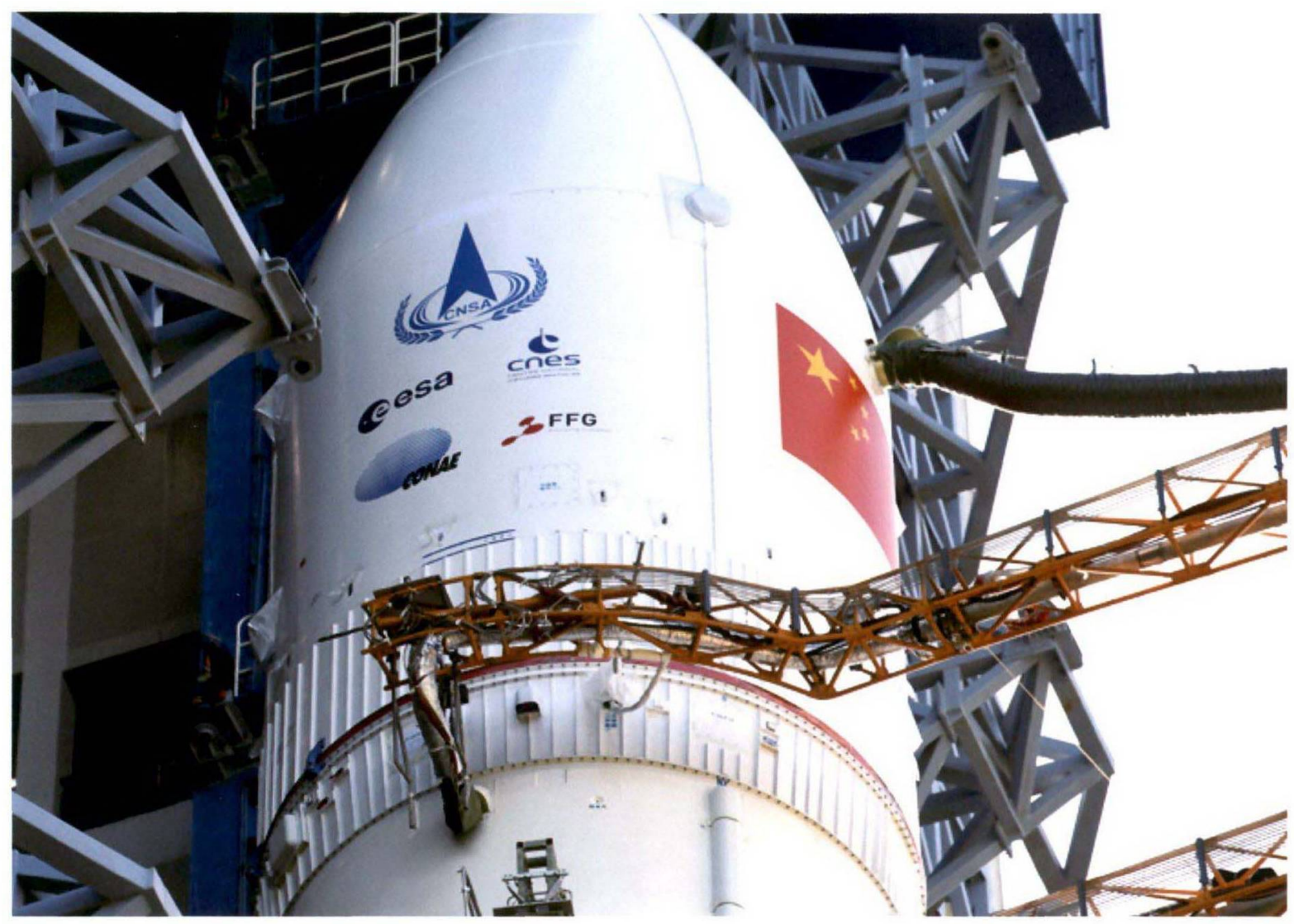

Tianwen-1 ready for launching (Image credit: CNSA)

aiming at the ultimate goal to explore and utilize space peacefully for the whole humankind. During the program's implementation, the Academy takes charge of the ground application system, the payload subsystem. the very long-baseline interferometry (VLBI) system for orbit measurement and determination. and the ReD of some important components. Joining hands with the China National Space Administration, the CAS will also conduct some applied research based on the scientific data obtained by the explorer.

A total of 13 institutions under CAS have committed to the R\&D activities as major contributors to the mission, represented by the National Astronomical Observatories and the National Space Science Center. The involved institutions also helped develop and synthesize some sophisticated aerospace materials, and remove some technological barriers that unless would have hampered the payload development and data processing. All these efforts have played an irreplaceable role in the smooth advancement of the mission.

Drawing on its long experiences in the implementation of Chang'e serial missions under China's Lunar Exploration Program. CAS has fostered and recruited a strong team of highly talented personnel for engineering management and technological development in a broad spectrum of areas for deep-space exploration. spanning from data reception. VLBI. light-weight payload development and important aerospace materials. With the rise of planetary science in China, a new generation of scientists have emerged at CAS institutes to explore scientific questions involved with Mars. It is expected that a series of discoveries might be made in life information, internal structure, geological characteristics and evolution of the planet. and also the characteristics. origin and evolution of its magnetic field.

\section{Reference}

W. X. Wan, C. Wang, C. L. Li, Y. Wei. (2020) China's first mission to Mars, Nature Astronomy, Vol. 4, 721 